\section{Korean Thyroid Imaging Reporting and Data System features of follicular thyroid adenoma and carcinoma: a single-center study}

\author{
Jung Won Park', Dong Wook Kim', Donghyun Kim', Jin Wook Baek', Yoo Jin Lee', \\ Hye Jin Baek ${ }^{2}$
}

\author{
'Department of Radiology, Busan Paik Hospital, Inje University College of Medicine, \\ Busan; ${ }^{2}$ Department of Radiology, Gyeongsang National University Changwon Hospital, \\ Gyeongsang National University School of Medicine, Changwon, Korea
}

Purpose: This study aimed to assess the diagnostic efficacy of Korean Thyroid Imaging Reporting and Data System (K-TIRADS) features for distinguishing follicular thyroid adenoma (FTA) from follicular thyroid carcinoma (FTC).

Methods: From January 2013 to July 2016, 46 follicular neoplasms in 45 patients who underwent preoperative thyroid ultrasonography (US) and thyroid surgery were included. The US features of each thyroid nodule were retrospectively evaluated by a single radiologist using a picture archiving and communication system. The diagnostic indices of K-TIRADS for follicular neoplasms were calculated according to whether K-TIRADS category 4 lesions were excluded or classified as benign or malignant.

Results: Of the 46 follicular neoplasms (mean size, $3.1 \pm 1.6 \mathrm{~cm}$ ), 37 were FTAs (mean size, $3.1 \pm 1.7$ $\mathrm{cm}$ ) and nine were FTCs (mean size, $3.0 \pm 1.5 \mathrm{~cm}$ ). A statistically significant difference was found between FTAs and FTCs regarding the margin $(P=0.035)$, while no significant differences were observed in the composition, echogenicity, shape, orientation, calcification, or vascularity of the lesions $(P<0.05)$. The FTAs belonged to K-TIRADS categories $3(n=22)$ and $4(n=15)$, while the FTCs belonged to K-TIRADS categories $3(n=4), 4(n=4)$, and $5(n=1)$. However, there was no statistically significant difference in the distribution of K-TIRADS categories between FTAs and FTCS $(P=0.184)$.

Conclusion: K-TIRADS features were not helpful for distinguishing FTA from FTC, although follicular neoplasms showed a high prevalence of K-TIRADS categories 3 and 4.

Keywords: Thyroid nodule; K-TIRADS; Thyroid cancer, follicular; Ultrasonography; Neoplasms

\section{Introduction}

Follicular thyroid adenoma (FTA) and carcinoma (FTC) are neoplasms associated with follicular cell

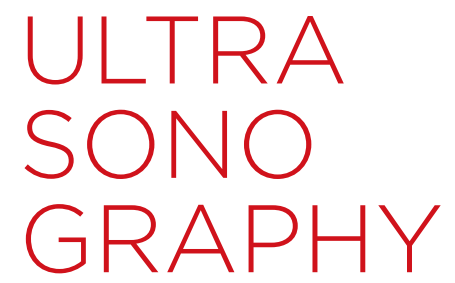

\section{ORIGINAL ARTICLE}

https://doi.org/10.14366/usg. 17020 pISSN: 2288-5919 - elSSN: 2288-5943 Ultrasonography 2017;36:349-354

Received: February 24, 2017

Revised: April 10, 2017

Accepted: April 13, 2017

Correspondence to: Dong Wook Kim, MD, Department of Radiology, Busan Paik Hospital, Inje University College of Medicine, 75 Bokji-ro, Busanjin-gu, Busan 47392, Korea

Tel. +82-51-890-6549

Fax. +82-51-896-1085

E-mail: dwultra@nate.com

This is an Open Access article distributed under the terms of the Creative Commons Attribution NonCommercial License (http://creativecommons.org/ licenses/by-nc/3.0//) which permits unrestricted noncommercial use, distribution, and reproduction in any medium, provided the original work is properly cited.

Copyright (C) 2017 Korean Society of Ultrasound in Medicine (KSUM)

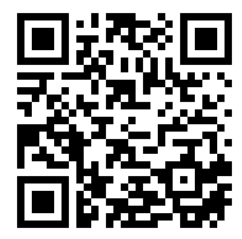

How to cite this article:

Park JW, Kim DW, Kim D, Baek JW, Lee YJ, Baek HJ. Korean Thyroid Imaging Reporting and Data System features of follicular thyroid adenoma and carcinoma: a single-center study. Ultrasonography. 2017 Oct;36(4):349354. 
differentiation [1]. It is difficult to distinguish FTA from FTC based on cytology alone; in fact, FTA and FTC should be differentiated on the basis of histopathology $[2,3]$. The histopathological features indicative of FTC include capsular invasion, vascular invasion, extrathyroidal tumor extension, lymph node metastases, and systemic metastases [1]. Vascular invasion, the most reliable sign of malignancy, refers to the penetration of the tumor into a vessel within or outside the capsule. To date, however, no specific ultrasonographic (US) features or classification system has been established for differentiating between FTA and FTC [4-6].

Recently, the Korean Society of Thyroid Radiology proposed a new risk stratification system for thyroid nodules, the Korean Thyroid Imaging Reporting and Data System (K-TIRADS) [5]. The algorithm used by K-TIRADS for malignancy risk stratification is based on the solidity and echogenicity of thyroid nodules. In a multicenter prospective validation study using K-TIRADS, the malignancy rates of category 2, 3, 4, and 5 nodules were $0.0 \%, 3.5 \%, 19.0 \%$, and $73.4 \%$, respectively [6]. However, no previous studies regarding the K-TIRADS categorization of FTA and FTC have been published, although their preoperative diagnosis is a long-standing dilemma. Therefore, the aim of this study was to assess the diagnostic efficacy of K-TIRADS features for distinguishing FTA from FTC.

\section{Materials and Methods}

\section{Patients}

This retrospective study was approved by the Institutional Review Board, and the need for informed consent was waived. From January 2013 to July 2016, 71 patients (62 women and 9 men; age range, 12 to 76 years; mean \pm standard deviation [SD], $48.5 \pm 15.1$ years) were diagnosed with a follicular neoplasm on the basis of surgical pathology following thyroidectomy. The exclusion criteria included the absence of preoperative thyroid US images $(n=19)$, thyroid US performed using an inappropriate protocol or poor image quality $(n=4)$, and an uncertain match between imaging findings and histopathological results $(n=3)$. Ultimately, 46 follicular neoplasms in 45 patients ( 37 women and 8 men; age range, 12 to 76 years; mean $\pm S D, 48.3 \pm 16.7$ years) were included in this study.

\section{Thyroid US}

Thyroid US was performed by two experienced radiologists using a high-resolution ultrasound instrument (iU 22, Philips Medical Systems, Bothell, WA, USA) equipped with a 5-12 MHz linear probe.

A single radiologist who had 15 years of experience in thyroid US examination, blinded to the condition (FTA or FTC), investigated the US features of each nodule on a picture archiving and communication system according to the K-TIRADS criteria [5]. The composition of a nodule was classified according to the ratio of the cystic portion in the entire nodule as follows: (1) solid (no obvious cystic component), (2) predominantly solid (cystic portion equal to, or less than, 50\%), (3) predominantly cystic (cystic portion greater than $50 \%$ ), or (4) cystic (no solid portion). The echogenicity of a nodule was classified as follows: (1) hypoechogenicity (hypoechoic relative to the thyroid parenchyma), (2) isoechogenicity (the same echogenicity as the thyroid parenchyma), or (3) hyperechogenicity (hyperechoic relative to the thyroid parenchyma). The shape of a nodule was classified as (1) round to oval or (2) irregular. The orientation of a nodule was classified as (1) parallel (anteroposterior diameter shorter than or equal to the transverse or longitudinal diameter) or (2) nonparallel (anteroposterior diameter longer than the transverse or longitudinal diameter in a transverse or longitudinal image). The margin of a nodule was classified as follows: (1) smooth (an obviously discernible smooth edge), (2) spiculated/microlobulated (an obviously discernible, but non-smooth edge), or (3) ill-defined (a poorly demarcated margin that could not be obviously differentiated from the adjacent thyroid tissue). Calcification was classified as follows: (1) microcalcification (tiny, punctate, echogenic foci of $1 \mathrm{~mm}$ or less, with or without posterior shadowing), (2) macrocalcification (echogenic foci larger than 1 $\mathrm{mm}$, with posterior acoustic shadowing), or (3) rim calcification (a peripheral curvilinear echogenic rim). Vascularity was classified as (1) none (no vascularity), (2) perinodular (circumferential vascularity), (3) mild intranodular (intranodular vascularity of less than $50 \%$ ), or (4) marked intranodular (intranodular vascularity of greater than $50 \%$ ). The presence of a hypoechoic halo, spongiform portion, and the comet-tail artifact was also investigated.

\section{Nodule Classification}

On the basis of the retrospective analysis of US features, each thyroid nodule was classified into K-TIRADS categories $2-5$ by the same radiologist [5]. Benign US features included spongiform, pure cysts, and partially cystic nodules with a comet tail artifact; suspicious features included microcalcification, a nonparallel orientation, and a spiculated or microlobulated margin. Thyroid nodules showing only benign US features were categorized as K-TIRADS category 2 (benign). Partially cystic and solid isoechoic or hyperechoic thyroid nodules without any suspicious US features were categorized as K-TIRADS category 3 (low suspicion). Partially cystic and solid isoechoic or hyperechoic thyroid nodules with any of the suspicious US features, and solid hypoechoic thyroid nodules without suspicious US features, were categorized as K-TIRADS category 4 (intermediate suspicion). Solid hypoechoic nodules with any of the suspicious US features were categorized as K-TIRADS category 5 (high suspicion). The diagnostic indices of K-TIRADS 
for follicular neoplasms were calculated depended on whether K-TIRADS category 4 was excluded or classified as benign or malignant.

\section{Statistical Analysis}

The normality of the distribution of the data was tested using the Kolmogorov-Smirnov test. According to the results of the Kolmogorov-Smirnov test, two numerical variables (age at the time of diagnosis and size of the nodule) showed a normal distribution $(P>0.05)$, and these variables were expressed as the mean $\pm S D$. Mean differences in age and the size of the nodule between the two groups (FTA vs. FTC) were compared using the independent $t$ test. Group comparisons of categorical variables were performed using the chi-square test or, for small cell values, the Fisher exact test. A receiver operating characteristic $(\mathrm{ROC})$ curve analysis was performed to evaluate the diagnostic performance of K-TIRADS, and the area under the ROC curve was compared between the two groups. All statistical analyses were performed using SPSS ver. 19.0 (IBM Corp., Armonk, NY, USA) or MedCalc ver. 9.0 (MedCalc Software, Mariakerke, Belgium), and $\mathrm{P}<0.05$ were considered to indicate statistical significance.

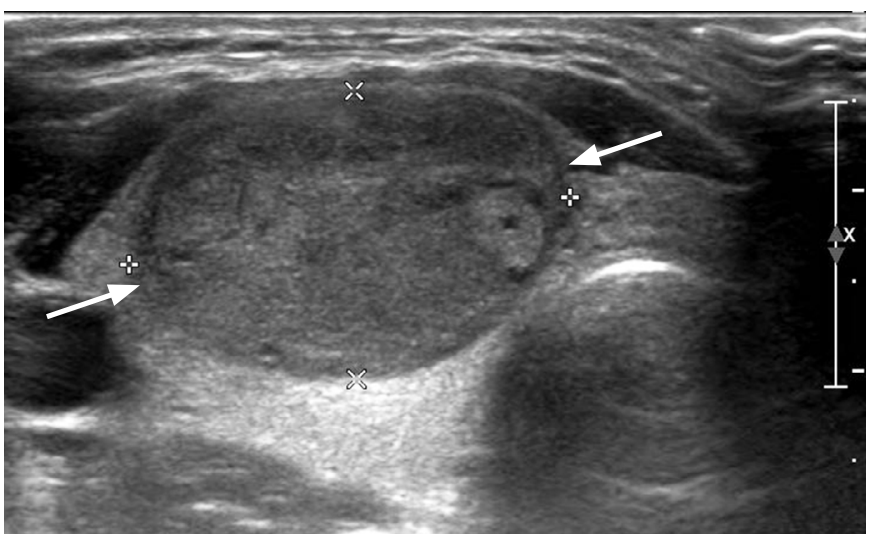

A

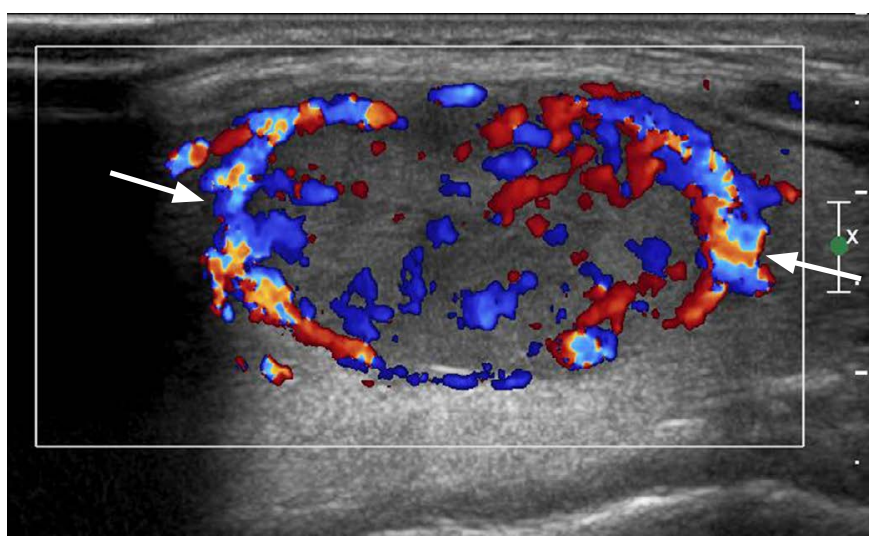

\section{Results}

Of the 46 follicular neoplasms, 37 (80.4\%) were FTAs (Fig. 1) and $9(19.6 \%)$ were FTCs (Fig. 2). The mean size of the follicular neoplasms was $3.1 \pm 1.6 \mathrm{~cm}$ (range, 0.7 to $9.5 \mathrm{~cm}$ ), and the mean size of FTAs and FTCs was $3.1 \pm 1.7 \mathrm{~cm}$ and $3.0 \pm 1.5 \mathrm{~cm}$, respectively. No statistically significant differences were found between FTAs and FTCs with regard to age $(P=0.151)$, sex $(P=0.679)$, nodular size $(P=0.882)$, or nodular location $(P=0.696)$.

The US features of FTA and FTC are summarized in Table 1. In both FTA and FTC, the halo sign, a spongiform appearance, and the comet-tail artifact were not found. A statistically significant difference was found regarding the margin $(P=0.035)$, while no significant differences were found in the composition, echogenicity, shape, orientation, calcification, or vascularity $(P>0.05)$.

The retrospective K-TIRADS analyses of FTA and FTC are presented in Table 2. The majority of FTAs and FTCs were classified as K-TIRADS category 3 (59.5\% [22 of 37] and $44.4 \%$ [4 of 9], respectively) or category $4(40.5 \%$ [ 15 of 37 ] and $44.4 \%$ [4 of 9], respectively). Only one case of FTC $(11.1 \%, 1$ of 9$)$ was considered to be category 5 . However, no statistically significant difference was

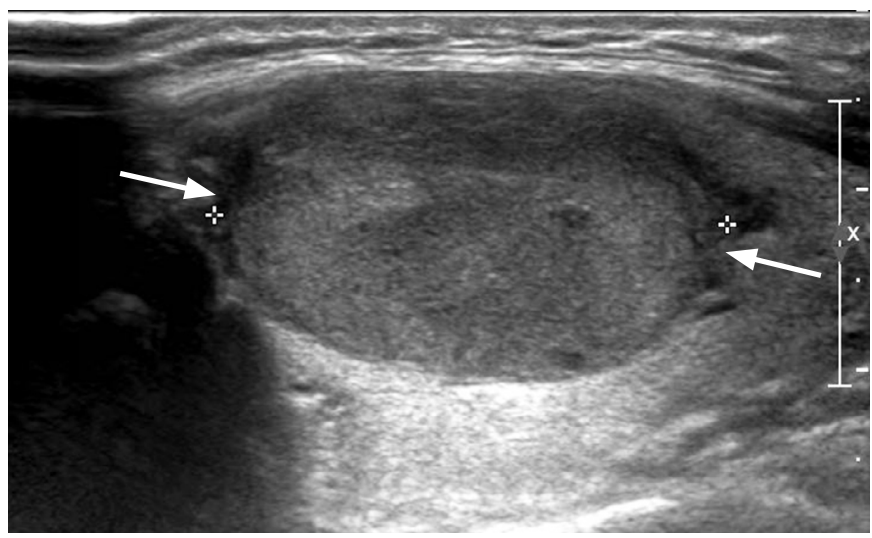

B

Fig. 1. Imaging results of a 25-year-old woman with follicular thyroid adenoma in the right lobe after hemithyroidectomy.

A, B. Transverse (A) and longitudinal (B) gray-scale ultrasonography show a solid thyroid nodule with hypoechogenicity, an oval shape with parallel orientation, and a smooth margin in the right thyroid lobe (arrows, $13.1 \mathrm{~mm} \times 16.3 \mathrm{~mm} \times 28.8 \mathrm{~mm}$ ). C. A longitudinal color Doppler ultrasonography shows marked intranodular vascularity (arrows). This nodule was categorized as Korean Thyroid Imaging Reporting and Data System category 4.

C 


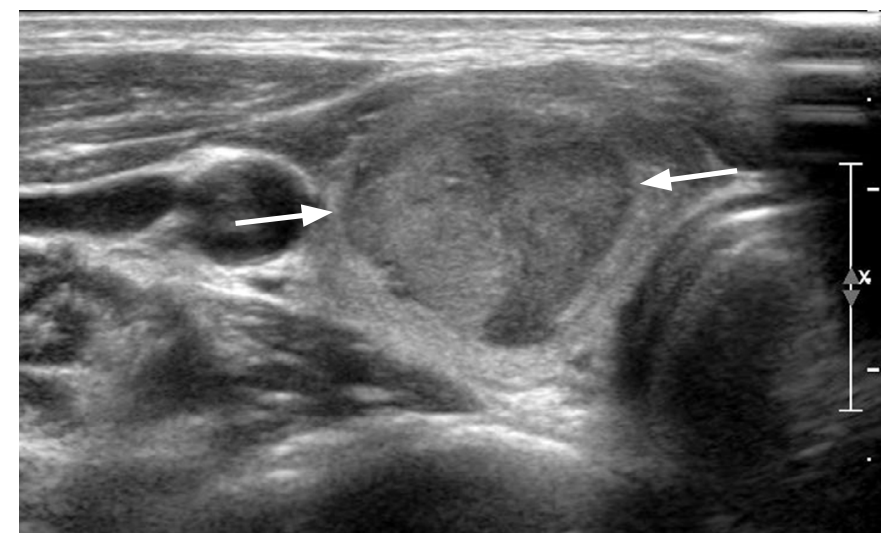

A

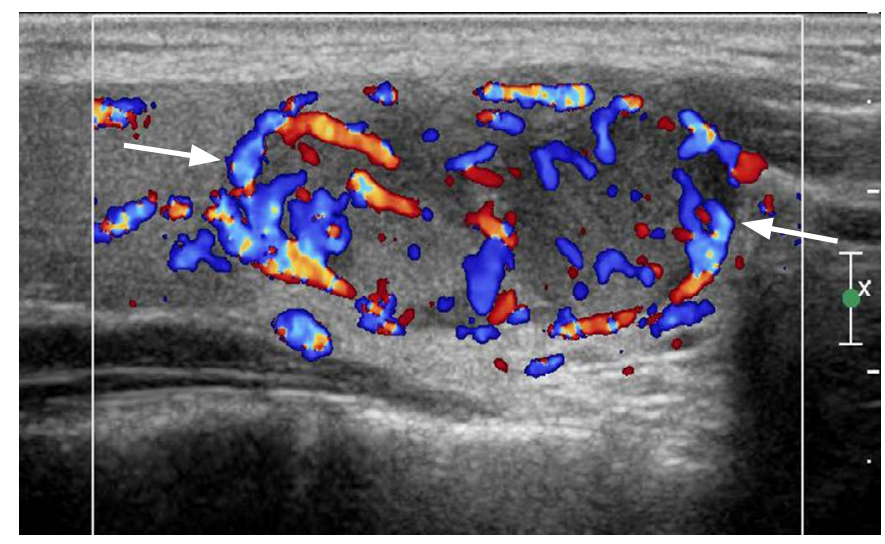

C

found in the distribution of K-TIRADS categories between FTA and FTC $(P=0.184)$. K-TIRADS category 4 cases were more likely to be malignant $(21.1 \%, 4$ of 19$)$ than K-TIRADS category 3 cases $(15.4 \%$, 4 of 26). However, no statistically significant difference was found in the risk of FTC between K-TIRADS category 3 and 4 cases ( $P=0.623$ ).

The diagnostic indices of K-TIRADS are presented in Table 3. When category 4 cases were excluded from the analysis, the diagnostic performance of K-TIRADS for differentiating FTC from FTA had the highest $A z$ value $(A z=0.600 ; 95 \% C l, 0.395$ to 0.782) with $a$ sensitivity of $20 \%$, a specificity of $100 \%$, a positive predictive value of $100 \%$, and a negative predictive value of $84.6 \%$. However, this result was not statistically significant $(P=0.371)$.

\section{Discussion}

According to previous studies, US is not helpful for differentiating FTA from FTC [1-5]. However, the possibility of a malignant follicular neoplasm may be greater in cases with a large nodule size, ill-defined margin, hypoechogenicity, thick irregular capsule, absence of a hypoechoic rim, presence of calcifications, and marked

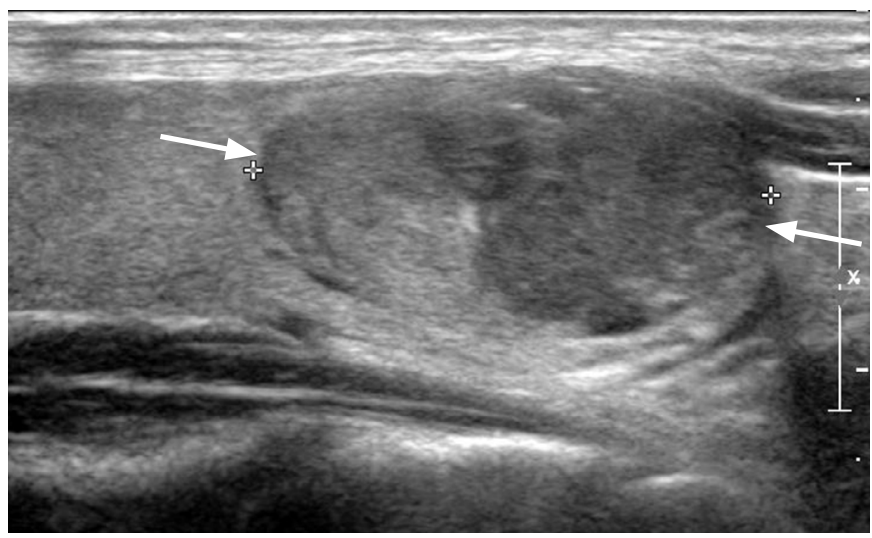

B

Fig. 2. Imaging results of a 30-year-old woman with follicular thyroid carcinoma in the right lobe after hemithyroidectomy.

A, B. Transverse (A) and longitudinal (B) gray-scale ultrasonography show a solid thyroid nodule with hypoechogenicity, an oval shape with parallel orientation, and a smooth margin in the right thyroid lobe (arrows, $16.0 \mathrm{~mm} \times 24.8 \mathrm{~mm} \times 28.5 \mathrm{~mm}$ ). C. A longitudinal color Doppler ultrasonography shows marked intranodular vascularity (arrows). The nodule was categorized as Korean Thyroid Imaging Reporting and Data System category 4.

intranodular vascularity [7-12]. In particular, the reliable US features of FTC include the invasion of adjacent vessels and extracapsular spread; however, these features are rare $[4,9]$. Recently, the K-TIRADS criteria have been introduced for the evaluation of thyroid nodules [5]. In the present study, we investigated the utility of K-TIRADS for distinguishing FTA from FTC. The majority of FTA and FTC cases were classified as K-TIRADS category 3 or 4 , and there was no significant difference in the distribution of K-TIRADS categories between FTA and FTC. Nevertheless, our study had several meaningful results. First, the majority of FTCs (88.9\%, 8 of 9) showed low- or intermediate-suspicion US features (K-TIRADS category 3 or 4), unlike papillary thyroid carcinoma, of which approximately $78.9 \%$ showed high-suspicion US features [6]. Second, follicular neoplasms never belonged to K-TIRADS category 2 in our study. Thus, a thyroid nodule belonging to K-TIRADS category 2 is unlikely to be a follicular neoplasm.

In previous studies $[6,13]$, the sensitivities of K-TIRADS categories 4 and 5 were found to be high ( $95.5 \%$ and $80.8 \%$, respectively). In the present study, the sensitivity of K-TIRADS categories 4 and 5 was only $55.6 \%$. The reason for this difference may be related to the fact 
Table 1. Frequency analysis of the ultrasonographic features of follicular thyroid adenoma and carcinoma

\begin{tabular}{|c|c|c|c|}
\hline Ultrasonographic feature & $\begin{array}{l}\text { Follicular thyroid } \\
\text { adenoma }(n=37)\end{array}$ & $\begin{array}{l}\text { Follicular thyroid } \\
\text { carcinoma }(n=9)\end{array}$ & P-value \\
\hline Composition & & & 0.707 \\
\hline Solid & $24(64.9)$ & $5(55.6)$ & \\
\hline Predominantly solid & $13(35.1)$ & $4(44.4)$ & \\
\hline Predominantly cystic & 0 & 0 & \\
\hline Cystic & 0 & 0 & \\
\hline Echogenicity & & & 0.317 \\
\hline Hypoechogenic & $18(48.6)$ & $6(66.7)$ & \\
\hline Isoechogenic & $19(51.4)$ & $3(33.3)$ & \\
\hline Hyperechogenic & 0 & 0 & \\
\hline Shape & & & 0.211 \\
\hline Round or oval & $29(78.4)$ & $5(55.6)$ & \\
\hline Irregular & $8(21.6)$ & $4(44.4)$ & \\
\hline Orientation & & & 1.000 \\
\hline Parallel & $36(97.3)$ & $9(100)$ & \\
\hline Nonparallel & $1(2.7)$ & 0 & \\
\hline Margin & & & 0.035 \\
\hline Smooth & $28(75.7)$ & $5(55.6)$ & \\
\hline Spiculated/Microlobulated & $2(5.4)$ & $3(33.3)$ & \\
\hline Ill-defined & $7(18.9)$ & $1(11.1)$ & \\
\hline Calcification & & & 0.734 \\
\hline None & $31(83.8)$ & $7(77.8)$ & \\
\hline Microcalcification & $1(2.7)$ & 0 & \\
\hline Macrocalcification & $1(2.7)$ & 0 & \\
\hline $\operatorname{Rim}$ & $4(10.8)$ & $2(22.2)$ & \\
\hline Vascularity & & & 0.835 \\
\hline None & $2(5.4)$ & 0 & \\
\hline Perinodular & $5(13.5)$ & $1(11.1)$ & \\
\hline Mild intranodular & $16(43.2)$ & $6(66.7)$ & \\
\hline Marked intranodular & $14(37.8)$ & $2(22.2)$ & \\
\hline \multicolumn{4}{|l|}{ Others } \\
\hline Halo & 0 & 0 & \\
\hline Spongiform & 0 & 0 & \\
\hline Comet-tail artifact & 0 & 0 & \\
\hline
\end{tabular}

Values are presented as number (\%). that our study included only FTA and FTC, and that the number of category 5 cases was very low $(n=1)$. Furthermore, the proportions of FTCs among malignant thyroid tumors in previous studies were low $(1.5 \%$ and $10.6 \%$, respectively) $[6,13]$. However, our study included 37 FTAs and nine FTCs, and we believe that this number of cases is appropriate for the comparison of the diagnostic indices of K-TIRADS between FTA and FTC. In the present study, K-TIRADS exhibited low diagnostic indices in differentiating between FTA and FTC.

According to a previous study, an ill-defined margin may be a likely indicator of malignancy in the case of follicular neoplasms [4]. Our study showed a statistically significant difference in the tumor margin between FTA and FTC. However, the ratio of ill-defined margins was similar between FTA and FTC, although FTC did show a higher rate of spiculated/microlobulated margins. Other US features, such as echogenicity, the presence and type of calcifications, and vascularity showed no statistically significant differences in the present study.

This study has several limitations. First, the sample size of follicular neoplasms in general was small, and this was especially true for FTCS. Second, all the US features of FTA and FTC were evaluated retrospectively. Third, a single radiologist performed the image analysis. Finally, while all US examinations were performed by two radiologists, we did not investigate interobserver variability.

In conclusion, the present study demonstrated that the majority of follicular neoplasms qualified as K-TIRADS category 3 or 4 . However, K-TIRADS features were not helpful for distinguishing between FTA

Table 2. K-TIRADS categories of follicular thyroid adenoma and carcinoma

\begin{tabular}{ccc}
\hline K-TIRADS (category) & $\begin{array}{c}\text { Follicular thyroid } \\
\text { adenoma }(n=37)\end{array}$ & $\begin{array}{c}\text { Follicular thyroid } \\
\text { carcinoma }(n=9)\end{array}$ \\
\hline 2 & 0 & 0 \\
3 & $22(59.5)$ & $4(44.4)$ \\
4 & $15(40.5)$ & $4(44.4)$ \\
5 & 0 & $1(11.1)$ \\
\hline
\end{tabular}

Values are presented as number (\%).

K-TIRADS, Korean Thyroid Imaging Reporting and Data System.

Table 3. Diagnostic indices of K-TIRADS categories for follicular thyroid adenoma and carcinoma

\begin{tabular}{|c|c|c|c|c|c|c|}
\hline K-TIRADS & Azvalue $e^{a)}$ & Sensitivity (\%) & Specificity (\%) & PPV (\%) & NPV (\%) & P-value \\
\hline When category 4 cases were excluded & $0.600(0.395-0.782)$ & 20 & 100 & 100 & 84.6 & 0.317 \\
\hline When category 4 cases were classified as benign & $0.556(0.402-0.702)$ & 11 & 100 & 100 & 82.2 & 0.313 \\
\hline When category 4 cases were classified as malignant & $0.575(0.421-0.719)$ & 55.6 & 59.5 & 25 & 84.6 & 0.439 \\
\hline
\end{tabular}

Az indicates the largest area under the receiver operating characteristic curve.

K-TIRADS, Korean Thyroid Imaging Reporting and Data System; PPV, positive predictive value; NPV, negative predictive value.

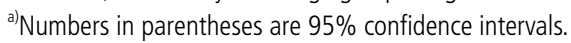


and FTC.

ORCID: Dong Wook Kim: http://orcid.org/0000-0002-9826-1326; Jin Wook Baek: http://orcid.org/0000-0003-4632-4951; Yoo Jin Lee: http://orcid.org/0000-00034701-7339; Hye Jin Baek: http://orcid.org/0000-0001-7349-2841

\section{Conflict of Interest}

No potential conflict of interest relevant to this article was reported.

\section{References}

1. McHenry CR, Phitayakorn R. Follicular adenoma and carcinoma of the thyroid gland. Oncologist 2011;16:585-593.

2. Mazzaferri EL. Management of a solitary thyroid nodule. N Engl J Med 1993;328:553-559.

3. Gharib $H$, Goellner JR. Fine-needle aspiration biopsy of the thyroid: an appraisal. Ann Intern Med 1993;118:282-289.

4. Wong KT, Ahuja AT. Ultrasound of thyroid cancer. Cancer Imaging 2005;5:157-166.

5. Shin JH, Baek JH, Chung J, Ha EJ, Kim JH, Lee YH, et al. Ultrasonography diagnosis and imaging-based management of thyroid nodules: revised Korean Society of Thyroid Radiology consensus statement and recommendations. Korean J Radiol 2016;17:370-395.

6. Ha EJ, Moon WJ, Na DG, Lee YH, Choi N, Kim SJ, et al. A multicenter prospective validation study for the Korean Thyroid Imaging Reporting and Data System in patients with thyroid nodules.
Korean J Radiol 2016;17:811-821.

7. Zhang JZ, Hu B. Sonographic features of thyroid follicular carcinoma in comparison with thyroid follicular adenoma. J Ultrasound Med 2014;33:221-227.

8. Sillery JC, Reading CC, Charboneau JW, Henrichsen TL, Hay ID, Mandrekar JN. Thyroid follicular carcinoma: sonographic features of 50 cases. AJR Am J Roentgenol 2010;194:44-54.

9. Kobayashi K, Hirokawa M, Yabuta T, Masuoka H, Fukushima M, Kihara $\mathrm{M}$, et al. Tumor protrusion with intensive blood signals on ultrasonography is a strongly suggestive finding of follicular thyroid carcinoma. Med Ultrason 2016;18:25-29.

10. lared W, Shigueoka DC, Cristofoli JC, Andriolo R, Atallah AN, Ajzen $S A$, et al. Use of color Doppler ultrasonography for the prediction of malignancy in follicular thyroid neoplasms: systematic review and meta-analysis. J Ultrasound Med 2010;29:419-425.

11. Song YS, Kim JH, Na DG, Min HS, Won JK, Yun TJ, et al. Ultrasonographic differentiation between nodular hyperplasia and neoplastic follicular-patterned lesions of the thyroid gland. Ultrasound Med Biol 2016;42:1816-1824.

12. Paramo JC, Mesko T. Age, tumor size, and in-office ultrasonography are predictive parameters of malignancy in follicular neoplasms of the thyroid. Endocr Pract 2008;14:447-451.

13. Na DG, Baek JH, Sung JY, Kim JH, Kim JK, Choi YJ, et al. Thyroid Imaging Reporting and Data System risk stratification of thyroid nodules: categorization based on solidity and echogenicity. Thyroid 2016;26:562-572. 